\title{
Numerical Solution of Systems of Ordinary Differential Equations With the Tau Method: An Error Analysis
}

\author{
By J. H. Freilich and E. L. Ortiz
}

\begin{abstract}
The recursive formulation of the Tau method is extended to the case of systems of ordinary differential equations, and an error analysis is given.

Upper and lower error bounds are given in one of the examples considered. The asymptotic behavior of the error compares in this case with that of the best approximant by algebraic polynomials for each of the components of the vector solution.
\end{abstract}

1. Introduction. Interest in the Tau method (see [2], [3], [5]), for a long time regarded only as a tool for the construction of accurate approximations of a very restricted class of functions, has been enhanced by the availability of software for its computer implementation and by the possibility of using it in the numerical solution of complex nonlinear differential equations over extended intervals. The approximation of the solution of such type of equations is achieved as a result of finding Tau approximants of a sequence of problems defined by linear differential equations. Details of this technique are given in [6].

The subject of this paper is the extension of Ortiz' recursive formulation of Lanczos' Tau method [5] to the case of systems of differential equations and, more particularly, to its error analysis for such systems.

Our error estimation technique is applied to three model examples for which the exact solution is readily available. It is discussed in general and with more detail when applied to the first of these examples. For the second example we show how to get upper and lower error bounds; we then compare these bounds with those given by Meinardus [4] for the best uniform approximation of each of the components of the vector solution by algebraic polynomials, to find that they are asymptotically equivalent. The third example is a differential equation with variable coefficients and a nonempty subspace of residuals; see [5].

Results of numerical experiments on the use of the Tau method for the approximate solution of systems of ordinary differential equations, with particular reference to stiff systems, are reported in [8]. The problems discussed in this paper can also be considered in the framework of simultaneous approximation of a function and its derivatives with the Tau method; see [1].

Received September 23, 1981.

1980 Mathematics Subject Classification. Primary 65L05, 65L10, 65N35.

Key words and phrases. Initial value problems, boundary value problems, systems of ordinary differential equations, simultaneous approximation of functions, Tau method, collocation methods. 


\section{Recursive Formulation of the Tau Method for Systems of Ordinary Differential} Equations. Without loss of generality we shall consider systems of order two. Let us consider the system which is equivalent to the general second order differential equation with variable coefficients

$$
\begin{aligned}
& y^{\prime \prime}(x)+a_{1}(x) y^{\prime}(x)+a_{0}(x) y(x)=0, \\
& y(0)=A, y^{\prime}(0)=B, 0 \leqslant x \leqslant r<\infty,
\end{aligned}
$$

where $a_{0}(x)$ and $a_{1}(x)$ are polynomials or sufficiently close uniform approximants of given functions by polynomials. Such approximations can be derived by using the Tau method itself, that is the approach followed in practical applications. If we set $z(x)=-y^{\prime}(x)$, (1) may be reposed as a system of first order differential equations which, in matrix form is

$$
D \mathbf{y}(x)=\left[\begin{array}{cc}
a_{0}(x) & -d / d x-a_{1}(x) \\
d / d x & 1
\end{array}\right]\left[\begin{array}{l}
y \\
z
\end{array}\right]=\left[\begin{array}{l}
0 \\
0
\end{array}\right] ; \quad \mathbf{y}(0)=\left[\begin{array}{c}
A \\
-B
\end{array}\right] .
$$

For the matrix operator $D$ we introduce a sequence of canonical polynomials $\mathbf{Q}=\left\{\mathbf{Q}_{n}(x)\right\}$, where each element $\mathbf{Q}_{n}(x)=\left\{\mathbf{Q}_{n}^{(1)}(x), \mathbf{Q}_{n}^{(2)}(x)\right\}$ is a vector such that

$$
D \mathbf{Q}_{n}^{(1)}(x)=\left[\begin{array}{c}
x^{n} \\
0
\end{array}\right]+\mathbf{R}_{n}^{(1)}(x) \text { and } D \mathbf{Q}_{n}^{(2)}=\left[\begin{array}{c}
0 \\
x^{n}
\end{array}\right]+\mathbf{R}_{n}^{(2)}(x)
$$

$\mathbf{R}_{n}^{(1)}(x), \mathbf{R}_{n}^{(2)}(x) \subset \mathbf{R}_{S}$, the subspace of residual vectors associated with $D$. If no gaps exist in the sequence $\mathbf{Q}$, then $\mathbf{R}_{S} \equiv \varnothing$. It is easy to verify that the properties of canonical polynomials discussed in Theorems 3.1-3.3 of Ortiz [5] are also valid in the vectorial case. From the point of view of the effective construction of approximate solutions of systems of ordinary differential equations with the Tau method, the fact that there exist a simple recursive relation between the vector canonical polynomials of $Q$ is of importance. Such a self-starting recursive relation is constructed, as in the case of one variable, on the basis of generating polynomials; see [5]. In the case $a_{0}(x)=1 / x^{2}, a_{1}(x)=1 / x$, they have the following form:

$$
D\left[\begin{array}{c}
x^{n} \\
0
\end{array}\right]=\left[\begin{array}{c}
x^{n-2} \\
n x^{n-1}
\end{array}\right], \quad D\left[\begin{array}{c}
0 \\
x^{n}
\end{array}\right]=\left[\begin{array}{c}
-(n+1) x^{n-1} \\
x^{n}
\end{array}\right] .
$$

Thus, for $n \geqslant 0$,

$$
\mathbf{Q}_{n}^{(1)}(x)=\frac{1}{\left[1+(2+n)^{2}\right]}\left[\begin{array}{c}
x^{n+2} \\
-(n+2) x^{n+1}
\end{array}\right]
$$

and

$$
\mathbf{Q}_{n}^{(2)}(x)=\frac{1}{\left[1+(1+n)^{2}\right]}\left[\begin{array}{c}
(n+1) x^{n+1} \\
x^{n}
\end{array}\right] .
$$

We would be interested in the solution $y$ on some compact interval $J$, say $J=[0,1]$, to the system (2). If the recursive formulation of the Tau method is used to find an approximate solution, it will have the form of a pair of polynomials: $\mathbf{y}_{n}(x)=$ $\left[y_{n}(x), z_{n}(x)\right]^{T}$, which solve exactly the perturbed system

$$
D \mathbf{y}_{n}(x)=\mathbf{H}_{n}(x)=\left[\tau_{1}^{(n)} \mathbf{H}_{n}^{(1)}(x), \tau_{2}^{(n)} \mathbf{H}_{n}^{(2)}(x)\right]^{T},
$$


where $\tau_{i}^{(n)} \mathbf{H}_{n}^{(i)}(x), i=1,2$, is usually a linear combination of Chebyshev or Legendre polynomials. The parameters $\tau_{i}^{(n)}$ are fixed so that the supplementary conditions

$$
\mathbf{y}_{n}(0)=[A,-B]^{T}
$$

are satisfied exactly.

The choice of the shifted Chebyshev polynomials in the right-hand side of (3) implies that the image $D \mathbf{e}_{n}$ of the error $\mathbf{e}_{n}=\mathbf{y}_{n}-\mathbf{y}$ has a balancing behavior in each of its components.

The error vector may be measured by any $1_{p}$ sum of the individual $1_{p}$ norms of its components, for $1 \leqslant p \leqslant \infty$. However, our interest lies in the double or vectorial uniform norm. For $J=[0,1]$ and $\mathbf{H}_{n}^{(i)}(x)=T_{n}^{*}(x), i=1,2$,

$$
\begin{aligned}
\left\|D \mathbf{e}_{n}\right\|_{\infty} & =\max \left\{\left\|\tau_{1}^{(n)} T_{n}^{*}(x)\right\|_{\infty},\left\|\tau_{2}^{(n)} T_{n}^{*}(x)\right\|_{\infty}\right\} \\
& \leqslant 2^{1-2 n} \max \left\{\left|\tau_{1}^{(n)}\right|,\left|\tau_{2}^{(n)}\right|\right\} .
\end{aligned}
$$

Now $T_{n}^{*}(x)=c_{0}^{(n)}+c_{1}^{(n)} x+\cdots+c_{n}^{(n)} x^{n}$, where the coefficients $c_{k}^{(n)}, k=0(1) n$, are available. Hence, $y_{n}$ will be of the form

$$
\mathbf{y}_{n}=\left[y_{n}, z_{n}\right]^{T}=\tau_{1}^{(n)} \sum_{k=0}^{n} c_{k}^{(n)} \mathbf{Q}_{k}^{(1)}(x)+\tau_{2}^{(n)} \sum_{k=0}^{n} c_{k}^{(n)} \mathbf{Q}_{k}^{(2)}(x) .
$$

If we set $\tau^{(n)}=\left[\tau_{1}^{(n)}, \tau_{2}^{(n)}\right]^{T}$, and $T_{n}^{*}(\mathbf{Q})=c_{0}^{(n)} \mathbf{Q}_{0}+\cdots+c_{n}^{(n)} \mathbf{Q}_{n}(x)$, we can represent the approximating vector solutions by $\mathbf{y}_{n}=\tau^{(n)} T_{n}^{*}(\mathbf{Q})$. The form of the solution when $\mathbf{R}_{S} \neq \varnothing$ follows from the argument given by Ortiz in [5]. An example is discussed in Section 4. The independence of the vector canonical polynomials from the interval $J$, in which the solution is sought, makes it possible to apply to systems the step by step technique discussed by Ortiz in [7] for the case of a single equation. If the steps are of constant length $h$, the same expression $\mathbf{y}_{n}$ will be needed in each step: only the $\tau$-terms will require updating. In both cases we could say that the integration formula used in each step is specifically designed for the given operator $D$ by our Tau technique.

3. The Case of Constant Coefficients. With the perturbed system (3) we are computing the exact solution $\left[y_{n}, z_{n}\right]^{T}$ of

$$
\left\{\begin{array}{l}
a_{0} y_{n}(x)-z_{n}^{\prime}(x)-a_{1} z_{n}(x)=\tau_{1}^{(n)} T_{n}^{*}(x), \\
y_{n}^{\prime}(x)+z_{n}(x)=\tau_{2}^{(n)} T_{n}^{*}(x)
\end{array}\right.
$$

with the original and derived initial conditions $y_{n}(0)=A, z_{n}(0)=-B$,

$$
y_{n}^{\prime}(0)=\tau_{2}^{(n)} T_{n}^{*}(0)+B ; \quad z_{n}^{\prime}(0)=-\tau_{1}^{(n)} T_{n}^{*}(0)+a_{0} A+a_{1} B .
$$

From (5)

$$
y_{n}^{\prime \prime}(x)+a_{1} y_{n}^{\prime}(x)+a_{0} y_{n}(x)=\tau_{1}^{(n)} T_{n}^{*}(x)+\tau_{2}^{(n)}\left[a_{1} T_{n}^{*}(x)+T_{n}^{*^{\prime}}(x)\right] .
$$

By repeated differentiation and back substitution, we find, assuming $A=1, B=0$, and $n=4$,

$$
\begin{aligned}
a_{0}^{3} a_{1}= & \tau_{1}^{(4)}\left[a_{0}^{2} a_{1} T_{4}^{*}(0)-a_{0}^{2} T_{4}^{*^{\prime}}(0)+a_{0} T_{4}^{*^{\prime \prime \prime}}(0)-a_{1} T_{4}^{*^{\prime \prime \prime \prime}}(0)\right] \\
& +\tau_{2}^{(4)}\left[a_{0}^{3} T_{4}^{*}(0)-a_{0}^{2} T_{4}^{*^{\prime \prime}}(0)+a_{0} a_{1} T_{4}^{*^{\prime \prime \prime}}(0)+\left[a_{0}-a_{1}^{2}\right] T_{4}^{*^{\prime \prime \prime \prime}}(0)\right] .
\end{aligned}
$$


Likewise, from

$$
z_{n}^{\prime \prime}(x)+a_{1} z_{n}^{\prime}(x)+a_{0} z_{n}(x)=\tau_{2}^{(n)} a_{0} T_{n}^{*}(x)-\tau_{1}^{(n)} T_{n}^{*^{\prime}}(x),
$$

we obtain

$$
\begin{aligned}
\left(a_{0}^{2} a_{1}^{2}-a_{0}^{3}\right)= & \tau_{1}^{(4)}\left[\left(a_{0} a_{1}^{2}-a_{0}^{2}\right) T_{4}^{*}(0)-a_{0} a_{1} T_{4}^{*^{\prime}}(0)+a_{0} T_{4}^{*^{\prime \prime}}(0)-T_{4}^{*^{\prime \prime \prime \prime}}(0)\right] \\
& +\tau_{2}^{(4)}\left[a_{0}^{2} a_{1} T_{4}^{*}(0)-a_{0}^{2} T_{4}^{*^{\prime}}(0)+a_{0} T_{4}^{*^{\prime \prime \prime}}(0)-a_{1} T_{4}^{*^{\prime \prime \prime \prime}}(0)\right] .
\end{aligned}
$$

We solve these two equations for $\tau_{1}^{(4)}$ and $\tau_{2}^{(4)}$. In Table I we illustrate the result for particular values of $a_{0}$ and $a_{1}$.

\section{TABLE I}

Evaluation of the Tau parameter for certain values of $a_{0}, a_{1}(n=4)$

\begin{tabular}{|c|c|c|c|c|}
\hline Example & $a_{0}$ & $a_{1}$ & $\tau_{1}^{(4)}$ & \multicolumn{1}{c|}{$\tau_{2}^{(4)}$} \\
\hline 1 & -1 & 1 & $-7.7667 \times 10^{-4}$ & $4.3971 \times 10^{-4}$ \\
2 & -1 & -1 & $-3.8373 \times 10^{-3}$ & $-2.3499 \times 10^{-3}$ \\
\hline
\end{tabular}

For a general prescription for $\tau_{1}^{(n)}, \tau_{2}^{(n)}$ we take the $r$ th derivative of Eq. (7), $r=0,1, \ldots, n$, and multiply through by $\left(1 / \lambda_{i}\right)^{r+1}, i=1,2$, where $\lambda_{1}, \lambda_{2}$ are distinct roots of the characteristic equation; they may be complex conjugates.

With each system $i=1,2$, we add all the equations together, making use of the fact that $\left(1 / \lambda_{i}\right)^{r+1}\left[1+a_{1}\left(1 / \lambda_{i}\right)+a_{0}\left(1 / \lambda_{i}\right)^{2}\right]=0$ to reduce the system.

Subtracting one system from the other and setting

$$
\begin{aligned}
& S_{\mathrm{e}}=\left(\lambda_{2}-\lambda_{1}\right)^{-1} \sum_{r=0}^{n}\left[\left(1 / \lambda_{1}\right)^{r+1}-\left(1 / \lambda_{2}\right)^{r+1}\right] T_{n}^{*^{(r)}}(0), \\
& S_{\mathrm{o}}=\left(\lambda_{2}-\lambda_{1}\right)^{-1} \sum_{r=1}^{n}\left[\left(1 / \lambda_{1}\right)^{r}-\left(1 / \lambda_{2}\right)^{r}\right] T_{n}^{*^{(r)}}(0),
\end{aligned}
$$

we find that $y_{n}(0)=\tau_{1}^{(n)} S_{\mathrm{e}}+\tau_{2}^{(n)}\left[a_{1} S_{\mathrm{e}}+S_{\mathrm{o}}\right]$. (Note: $S_{\mathrm{e}}$ and $S_{\mathrm{o}}$ are real.) Similarly, from (8) $z_{n}(0)=\tau_{2}^{(n)} a_{0} S_{\mathrm{e}}-\tau_{1}^{(n)} S_{\mathrm{o}}$.

When $A=1, B=0$, the above equations yield

$$
\tau_{1}^{(n)}=a_{0} S_{\mathrm{e}} /\left(S_{\mathrm{o}}^{2}+a_{1} S_{\mathrm{o}} S_{\mathrm{e}}+a_{\mathrm{o}} S_{\mathrm{e}}^{2}\right) ; \quad \tau_{2}^{(n)}=S_{\mathrm{o}} /\left(S_{\mathrm{o}}^{2}+a_{1} S_{\mathrm{o}} S_{\mathrm{e}}+a_{0} S_{\mathrm{e}}^{2}\right) .
$$

We shall use the following expansion for $T_{n}^{*}(x)$ :

$$
T_{n}^{*}(x)=\frac{1}{2}\left[(4 x)^{n}+\sum_{j=1}^{n-1}(-1)^{j}\left(\begin{array}{c}
2 n-j-1 \\
j
\end{array}\right) \frac{n}{n-j}(4 x)^{n-j}+2(-1)^{n}\right] .
$$

Hence $T_{n}^{*^{(n)}}(0)=2^{2 n-1} n ! ; T_{n}^{*^{(n-1)}}(0)=2^{2 n-1} n !\left(-\frac{1}{2}\right)$. For $2 \leqslant k \leqslant n$ we have

$$
\begin{aligned}
& T_{n}^{*^{(n-k)}}(0)= 2^{2 n-1} n !\left[\frac{(-1)^{k} 2^{-2 k} 2 n(2 n-k-1) \cdots(2 n-2 k+1)}{k ! n(n-1) \cdots(n-k+1)}\right] \\
&= 2^{2 n-1} n !\left[\frac{(-1)^{k} 2^{-k}}{k !}\left(1-\frac{k-1}{2(n-1)}\right)\left(1-\frac{k-2}{2(n-2)}\right)\right. \\
&\left.\cdots\left(1-\frac{1}{2(n-k+1)}\right)\right] .
\end{aligned}
$$


THEOREM 1. Suppose $\lambda_{2}^{t}-\lambda_{1}^{t} \neq 0$ for $t=r$ and $t=p(r), 0<t \leqslant n+1$, where $p(r)$ is the first preceding integer to $r$ satisfying the stated inequality. Necessarily $p(r)$ is either $r-1$ or $r-2$. Then if

$$
1 \geqslant\left|\frac{\lambda_{2}^{r}-\lambda_{1}^{r}}{\lambda_{2}^{p(r)}-\lambda_{1}^{p(r)}}\right| \geqslant\left(\lambda_{1} \lambda_{2}\right)^{r-p(r)} \text { for } 1<r \leqslant n+1,
$$

we may deduce the following asymptotic results

$$
\begin{aligned}
& \lim _{n \rightarrow \infty}\left|\tau_{1}^{(n)}\right|=O(1) /\left|\lambda_{2}^{-p(n+2)}-\lambda_{1}^{-p(n+2)}\right| 2^{2 n-1} n !, \\
& \lim _{n \rightarrow \infty}\left|\tau_{2}^{(n)}\right|=O(1) /\left|\lambda_{2}^{-p(n+1)}-\lambda_{1}^{-p(n+1)}\right| 2^{2 n-1} n ! .
\end{aligned}
$$

Proof. From Eq. (12) we have that $\left|T_{n}^{*^{(r)}}(0)\right|<\frac{1}{2}\left|T_{n}^{*^{(r+1)}}(0)\right|$ for all $0<r<n-1$. On applying this inequality in (11), the result follows.

Let us assume that $\lambda_{1} \neq \lambda_{2}$ and that both are real. Then

$$
y(x)=c_{1} \exp \left(\lambda_{1} x\right)+c_{2} \exp \left(\lambda_{2} x\right), \quad z(x)=d_{1} \exp \left(\lambda_{1} x\right)+d_{2} \exp \left(\lambda_{2} x\right) .
$$

Set $k(x, t):=\exp \left(\lambda_{2} x+\lambda_{1} t\right)-\exp \left(\lambda_{1} x+\lambda_{2} t\right)$, and let $W(t)$ be the wronskian of the basis functions for the solution of our equation. Then

$$
\phi(x, t):=k(x, t) / W(t)=\left(\lambda_{2}-\lambda_{1}\right)^{-1}\left[\exp \left(\lambda_{2}(x-t)\right)-\exp \left(\lambda_{1}(x-t)\right)\right]
$$

and

$$
\frac{\partial}{\partial t} \phi(x, t)=\left(\lambda_{2}-\lambda_{1}\right)^{-1}\left[\lambda_{1} \exp \left(\lambda_{1}(x-t)\right)-\lambda_{2} \exp \left(\lambda_{2}(x-t)\right)\right],
$$

which is equal to -1 at $t=x$.

The solution of (7) has the form

$$
y_{n}(x)=C_{1} \exp \left(\lambda_{1} x\right)+C_{2} \exp \left(\lambda_{2} x\right)-\tau_{2}^{(n)} T_{n}^{*}(0) \phi(x, 0)+\int_{0}^{x} G_{1}(x, t) d t,
$$

with

$$
G_{1}(x, t)=\left[\left(\tau_{1}^{(n)}+a_{1} \tau_{2}^{(n)}\right) T_{n}^{*}(t) k(x, t)-\tau_{2}^{(n)} W(t) T_{n}^{*}(t) \frac{\partial}{\partial t} \phi(x, t)\right] / W(t) .
$$

Moreover,

$$
\begin{aligned}
y_{n}^{\prime}(x)= & C_{1} \lambda_{1} \exp \left(\lambda_{1}, x\right)+C_{2} \lambda_{2} \exp \left(\lambda_{2} x\right)-\tau_{2}^{(n)} T_{n}^{*}(0) \\
& \times\left[\lambda_{2} \exp \left(\lambda_{2} x\right)-\lambda_{1} \exp \left(\lambda_{1} x\right)\right] / W(0) \\
& +G_{1}(x, x)+\int_{0}^{x} \frac{\partial}{\partial x} G_{1}(x, t) d t
\end{aligned}
$$

Clearly $y_{n}(0)=C_{1}+C_{2}, y_{n}^{\prime}(0)=C_{1} \lambda_{1}+C_{2} \lambda_{2}$. Then

$$
C_{1}-c_{1}=-\tau_{2}^{(n)} T_{n}^{*}(0) /\left(\lambda_{2}-\lambda_{1}\right), \quad C_{2}-c_{2}=\tau_{2}^{(n)} T_{n}^{*}(0) /\left(\lambda_{2}-\lambda_{1}\right) .
$$

We set

$$
g_{i}(x):=\int_{0}^{x} T_{n}^{*}(t)\left[\frac{\partial^{i-1}}{\partial t^{i-1}} \phi(x, t)\right] d t, \quad i=1,2 .
$$

Then

$$
\begin{aligned}
& e_{1}^{(n)}(x):=y_{n}(x)-y(x)=\left(\tau_{1}^{(n)}+a_{1} \tau_{2}^{(n)}\right) g_{1}(x)-\tau_{2}^{(n)} g_{2}(x), \\
& e_{2}^{(n)}(x):=z_{n}(x)-z(x)=a_{0} \tau_{2}^{(n)} g_{1}(x)+\tau_{1}^{(n)} g_{2}(x) .
\end{aligned}
$$


THEOREM 2. Upper bounds for the error vector $\mathrm{e}^{(n)}=\left(e_{1}^{(n)}, e_{2}^{(n)}\right)^{T}$ :

$$
\begin{aligned}
\left\|e_{1}^{(n)}\right\|_{\infty} \leqslant & \frac{\left|\tau_{1}^{(n)}+a_{1} \tau_{2}^{(n)}\right|}{2(n-1)\left|\lambda_{1}-\lambda_{2}\right|}\left|\exp \left(\lambda_{1}\right)-\exp \left(\lambda_{2}\right)\right| \\
& +\frac{\left|\tau_{2}^{(n)}\right|}{2(n-1)}\left(\frac{\left|\lambda_{2} \exp \left(\lambda_{2}\right)-\lambda_{1} \exp \left(\lambda_{1}\right)\right|}{\left|\lambda_{2}-\lambda_{1}\right|}+2\right), \\
\left\|e_{2}^{(n)}\right\|_{\infty} \leqslant & \frac{\left|a_{0} \tau_{2}^{(n)}\right|}{2(n-1)\left|\lambda_{1}-\lambda_{2}\right|}\left|\exp \left(\lambda_{1}\right)-\exp \left(\lambda_{2}\right)\right| \\
& +\frac{\left|\tau_{1}^{(n)}\right|}{2(n-1)}\left(\frac{\left|\lambda_{2} \exp \left(\lambda_{2}\right)-\lambda_{1} \exp \left(\lambda_{1}\right)\right|}{\left|\lambda_{2}-\lambda_{1}\right|}+2\right) .
\end{aligned}
$$

Proof. We set

$$
I(t)=\int T_{n}^{*}(t) d t=\frac{1}{4}\left[\frac{T_{n+1}^{*}(t)}{n+1}-\frac{T_{n-1}^{*}(t)}{n-1}\right] .
$$

Then

(15) $|I(0)|=1 /\left(2\left(n^{2}-1\right)\right)$, while $|I(t)| \leqslant n /\left[2\left(n^{2}-1\right)\right]$, for $0 \leqslant t \leqslant 1$.

Integrating by parts in (13) and using these bounds, we find that for all $0 \leqslant x \leqslant 1$ :

$$
\left|g_{2}(x)\right| \leqslant \frac{\left.\left.|n| \frac{\partial}{\partial t} \phi(x, t)\right|_{t=x}+\left|\frac{\partial}{\partial t} \phi(x, t)\right|_{t=0}+n \int_{0}^{x}\left|\frac{\partial^{2}}{\partial t^{2}} \phi(x, t)\right| d t\right]}{\left[2\left(n^{2}-1\right)\right]},
$$

Using the positivity of $e^{\lambda t}, \lambda^{2} e^{\lambda t}$ and the convexity of $\lambda e^{\lambda x}, \lambda>0$, the result follows.

For $n=4$, with the same examples as in Table I, we obtain the following results:

TABLE II

Upper bounds for the components of the error vector

\begin{tabular}{|c|c|c|c|c|}
\hline Example & $g_{1}$ & $g_{2}$ & $e_{1}^{(n)}$ & $e_{2}^{(n)}$ \\
\hline 1 & 0.123505 & 0.442712 & $2.3629 \times 10^{-4}$ & $3.9815 \times 10^{-4}$ \\
2 & 0.335721 & 0.966375 & $2.7703 \times 10^{-3}$ & $4.4972 \times 10^{-3}$ \\
\hline
\end{tabular}

4. The Harmonic Oscillator. We will now refine the previous results for the case of the harmonic oscillator

$$
D \mathbf{y}(x)=\left[\begin{array}{cc}
1 & -\frac{d}{d x} \\
\frac{d}{d x} & 1
\end{array}\right]\left[\begin{array}{l}
y \\
z
\end{array}\right]=\left[\begin{array}{l}
0 \\
0
\end{array}\right]
$$

subject to $\mathbf{y}(0)=[1,0]^{T}$, for $0 \leqslant x \leqslant 1$. 
The canonical polynomials associated with (16) are generated with the technique sketched in Section 2. They are

$$
\begin{aligned}
& \mathbf{Q}_{n}^{(1)}(x)=\left[\begin{array}{c}
x^{n} \\
-n x^{n-1}
\end{array}\right]-n(n-1) \mathbf{Q}_{n-2}^{(1)}(x) \\
& \mathbf{Q}_{n}^{(2)}(x)=\left[\begin{array}{c}
n x^{n-1} \\
x^{n}
\end{array}\right]-n(n-1) \mathbf{Q}_{n-2}^{(2)}(x)
\end{aligned}
$$

that is,

$$
\mathbf{Q}_{0}^{(1)}(x)=\left[\begin{array}{l}
1 \\
0
\end{array}\right], \quad \mathbf{Q}_{1}^{(1)}(x)=\left[\begin{array}{c}
x \\
-1
\end{array}\right], \quad \mathbf{Q}_{2}^{(1)}(x)=\left[\begin{array}{c}
x^{2}-x \\
-2 x
\end{array}\right], \ldots
$$

and

$$
\mathbf{Q}_{0}^{(2)}(x)=\left[\begin{array}{l}
0 \\
1
\end{array}\right], \quad \mathbf{Q}_{1}^{(2)}(x)=\left[\begin{array}{l}
1 \\
x
\end{array}\right], \quad \mathbf{Q}_{2}^{(2)}(x)=\left[\begin{array}{c}
2 x \\
x^{2}-2
\end{array}\right], \ldots
$$

With them we construct the vector Tau simultaneous approximation $\mathbf{y}_{n}(x)=$ $\boldsymbol{\tau}^{(n)} T_{n}^{*}(\mathbf{Q})$. The Tau vector is determined with the help of the supplementary condition $\mathbf{y}_{n}(0)=\mathbf{y}(0)$. For $n=4$ we find (to $6 \mathrm{D}$ )

$$
\mathbf{y}_{4}(x)=\left[\begin{array}{c}
0.035808 x^{4}-0.006633 x^{3}-0.502305 x^{2}-0.000153 x+1 \\
0.019562 x^{4}-0.182355 x^{3}+0.004552 x^{2}+0.999720 x
\end{array}\right] \text {. }
$$

THEOREM 3. Upper and lower error bounds for the Tau vector $\tau^{(n)}$. For $n$ even

$$
\frac{0.83386}{2^{2 n-1} n !}<\left|\tau_{1}^{(n)}\right|<\frac{0.90227}{2^{2 n-1} n !}, \quad \frac{0.45648}{2^{2 n-1} n !}<\left|\tau_{2}^{(n)}\right|<\frac{0.49455}{2^{2 n-1} n !} .
$$

For $n$ odd the bounds should be interchanged.

Proof. From (9) and (10)

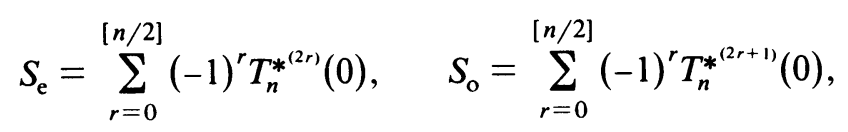

while (11) becomes $\tau_{1}^{(n)}=S_{\mathrm{e}} /\left(S_{\mathrm{e}}^{2}+S_{\mathrm{o}}^{2}\right), \tau_{2}^{(n)}=S_{\mathrm{o}} /\left(S_{\mathrm{e}}^{2}+S_{\mathrm{o}}^{2}\right)$. We can now apply (12) to deduce that for even $n$

$$
\begin{aligned}
1-\sum_{r=0}^{\infty} \frac{\left(\frac{1}{2}\right)^{4 r+2}}{(4 r+2) !}+\frac{\left(\frac{1}{2}\right)^{4}}{4 !} & {\left[\left(1-\frac{3}{2(n-1)}\right)\left(1-\frac{1}{(n-2)}\right)\left(1-\frac{1}{n-3}\right)\right] } \\
< & \frac{(-1)^{[n / 2]}}{2^{n-1} n !} S_{\mathrm{e}}<\sum_{r=0}^{\infty} \frac{\left(\frac{1}{2}\right)^{4 r}}{(4 r) !}-\frac{\left(\frac{1}{2}\right)^{2}}{2 !}\left(1-\frac{1}{2(n-1)}\right)
\end{aligned}
$$

and

$$
\begin{aligned}
\frac{1}{2}-\sum_{r=1}^{\infty} \frac{\left(\frac{1}{2}\right)^{4 r-1}}{(4 r-1) !} & <\frac{(-1)^{[n / 2]}}{2^{2 n-1} n !} S_{\mathrm{o}}<\sum_{r=0}^{\infty} \frac{\left(\frac{1}{2}\right)^{4 r+1}}{(4 r+1) !} \\
& -\frac{\left(\frac{1}{2}\right)^{3}}{3 !}\left[\left(1-\frac{2}{2(n-2)}\right)\left(1-\frac{1}{2(n-2)}\right)\right]
\end{aligned}
$$


If we take $n=4$ and make use of expansions for $\sin x, \sinh x ; \cos x$ and $\cosh x$, we obtain

$$
\begin{gathered}
1-\frac{\cosh \left(\frac{1}{2}\right)-\cos \left(\frac{1}{2}\right)}{2}+\frac{\left(\frac{1}{2}\right)^{7}}{4 !}<\frac{(-1)^{[n / 2]}}{2^{2 n-1} n !} S_{\mathrm{e}}<\frac{\cosh \left(\frac{1}{2}\right)+\cos \left(\frac{1}{2}\right)}{2}-\frac{5\left(\frac{1}{2}\right)^{2}}{2 ! 6} \\
\frac{1}{2}-\frac{\sinh \left(\frac{1}{2}\right)-\sin \left(\frac{1}{2}\right)}{2}<\frac{(-1)^{[n / 2]}}{2^{2 n-1} n !} S_{\mathrm{o}}<\frac{\sinh \left(\frac{1}{2}\right)+\sin \left(\frac{1}{2}\right)}{2}-\frac{\left(\frac{1}{2}\right)^{4}}{3 !}
\end{gathered}
$$

Hence,

$$
0.875304<\frac{(-1)^{[n / 2]}}{2^{2 n-1} n !} S_{\mathrm{e}}<0.898438
$$

and

$$
0.479165<\frac{(-1)^{[n / 2]}}{2^{2 n-1} n !} S_{\mathrm{o}}<0.492448
$$

Consequently,

$$
1.0497>\frac{S_{\mathrm{e}}^{2}+S_{\mathrm{o}}^{2}}{\left(2^{2 n-1} n !\right)^{2}}>0.9958
$$

Example. For $n=4$ we find

$2.714 \times 10^{-4}<\tau_{1}^{(4)}<2.937 \times 10^{-4}$ and $1.486 \times 10^{-4}<\tau_{2}^{(4)}<1.610 \times 10^{-4}$;

the computed values are $\tau_{1}^{(4)}=2.797 \times 10^{-4}$ and $\tau_{2}^{(4)}=1.528 \times 10^{-4}$. For the ratio of the Tau-terms our estimations give

$$
\tau_{1}^{(4)} / \tau_{2}^{(4)}<1.875
$$

while the computed value is 1.830 .

We shall require the following result, concerning the integral of a Chebyshev polynomial between two consecutive zeros, which is easy to derive.

LEMMA 1. Let the zeros of $T_{n}^{*}(x)$ be $x_{n-k}, k=1(1) n$, where

$$
x_{n-k}=\cos ^{2} \frac{\left(n-k+\frac{1}{2}\right) \pi}{2 n} \text { and } x_{n}=0<x_{n-1}<\cdots<x_{0}<1=x_{-1} \text {. }
$$

Then,

$$
I_{j}=\int_{x_{j}}^{x_{j-1}} T_{n}^{*}(t) d t=(-1)^{j} \phi(n) \sin \frac{j \pi}{n}, \text { for } j=1(1) n-1,
$$

where

$$
\phi(n)=\left[n /\left(n^{2}-1\right)\right] \cos \frac{\pi}{2 n} \rightarrow \frac{1}{n}\left[1+O\left(1 / n^{2}\right)\right]
$$

and

$$
I_{j}=\left[(-1)^{j} / 2\left(n^{2}-1\right)\right]\left[n \sin \frac{\pi}{2 n}-1\right] \text { for } j=0 \text { or } j=n
$$


We shall now restrict ourselves to the case of $n$ being even, as the treatment for $n$ odd is similar. We shall set $P(n)=\phi(n)(1+O(1 / n))$. For $a_{1}=0, a_{0}=1,(14)$ and (15) take the following form:

$$
\begin{aligned}
& e_{1}^{(n)}(x):=y_{n}(x)-y(x)=\int_{0}^{x}\left[\tau_{1}^{(n)} \sin (x-t)+\tau_{2}^{(n)} \cos (x-t)\right] T_{n}^{*}(t) d t \\
& e_{2}^{(n)}(x):=z_{n}(x)-z(x)=\int_{0}^{x}\left[\tau_{2}^{(n)} \sin (x-t)-\tau_{1}^{(n)} \cos (x-t)\right] T_{n}^{*}(t) d t
\end{aligned}
$$

THEOREM 4. Upper and lower bounds for the error vector $\mathbf{e}^{(n)}=\left[e_{1}^{(n)}, e_{2}^{(n)}\right]^{T}$.

(i) For the function component

$$
\frac{0.08518 P(n)}{2^{2 n} n !}<\left\|e_{1}^{(n)}\right\|<\frac{1.50220(1+O(1 / n))}{2^{2 n}(n+1) !} .
$$

(ii) For the derivative component

$$
\frac{0.49468 P(n)}{2^{2 n} n !}<\left\|e_{2}^{(n)}\right\|<\frac{1.76971(1+O(1 / n))}{2^{2 n}(n+1) !} .
$$

Proof. Part (i). To find an upper bound for $e_{1}^{(n)}$ we integrate by parts in (17).

$$
\begin{aligned}
e_{1}^{(n)}(x)= & \frac{\tau_{2}^{(n)}}{4}\left[\frac{T_{n+1}^{*}(x)}{n+1}-\frac{T_{n-1}^{*}(x)}{n-1}\right] \\
& -\frac{(-1)^{n}}{2\left(n^{2}-1\right)}\left(\tau_{1}^{(n)} \sin x+\tau_{2}^{(n)} \cos x\right) \\
& +\int_{0}^{x} I(t)\left[-\tau_{1}^{(n)} \cos (x-t)+\tau_{2}^{(n)} \sin (x-t)\right] d t
\end{aligned}
$$

By (15),

$$
\left|e_{1}^{(n)}(x)\right| \leqslant \frac{\left\{\tau_{2}^{(n)} n+\tau_{1}^{(n)} \sin x+\tau_{2}^{(n)} \cos x+\left[\tau_{2}^{(n)}(1-\cos x)+\tau_{1}^{(n)} \sin x\right] n\right\}}{2\left(n^{2}-1\right)}
$$

and

$$
\begin{aligned}
\left\|e_{1}^{(n)}\right\| & \leqslant\left[\left|\tau_{2}^{(n)}\right|(2-\cos (1))+\left|\tau_{1}^{(n)}\right| \sin (1)\right]\left[\frac{1}{2(n+1)}+O\left(1 / n^{2}\right)\right] \\
& \leqslant 1.5022(1+O(1 / n)) /\left[2^{2 n}(n+1) !\right] .
\end{aligned}
$$

To find a lower bound for $\left\|e_{1}^{(n)}\right\|$ we shall consider $e_{1}^{(n)}\left(x_{3 n / 4}\right)$ with $n$ an odd multiple of $4, n \geqslant 12$. Then

$$
\begin{aligned}
& x_{3 n / 4}=\frac{1}{2}[1+\cos (3 \pi / 4+\pi / 2 n)] \rightarrow \frac{1}{2}(1-1 / \sqrt{2}), \\
& \int_{0}^{x_{3 n / 4}} \cos \left(x_{3 n / 4}-t\right) T_{n}^{*}(t) d t \\
& >\cos x_{3 n / 4} I_{n}+\cos \left(x_{3 n / 4}-x_{n-2}\right)\left[I_{n-2}-\left|I_{n-1}\right|\right] \\
& +\cdots+\cos \left(x_{3 n / 4}-x_{3 n / 4+1}\right)\left[I_{3 n / 4+1}-\left|I_{3 n / 4+2}\right|\right] \\
& >\cos x_{3 n / 4}\left[I_{n}+\phi(n) \sum_{k=1}^{n / 4-1}(-1)^{k} \sin (k \pi / n)\right] \\
& >2 \cos x_{3 n / 4} \sin \pi / 2 n \phi(n) \sum_{k=1}^{(n-4) / 8} \cos 2 k \pi / n \text {. }
\end{aligned}
$$


But

$$
\sum_{k=1}^{(n-4) / 8} \cos 2 k \pi / n=\frac{\cos (2 \pi / n)-1}{2(1-\cos 2 \pi / n)}+\frac{\sin \pi / 4}{2 \sin \pi / n} \rightarrow(n / 2 \pi \sqrt{2})+O(1) .
$$

Hence, for large $n$

$$
\int_{0}^{x_{3 n / 4}} \cos \left(x_{3 n / 4}-t\right) T_{n}^{*}(t) d t>\cos [(2-\sqrt{2}) / 4] P(n) / 2 \sqrt{2} .
$$

Now

$$
\begin{aligned}
\int_{0}^{x_{3 n / 4}} \sin \left(x_{3 n / 4}-t\right) T_{n}^{*}(t) d t> & {\left[\sin \left(x_{3 n / 4}-x_{n-1}\right) I_{n-1}+\sin \left(x_{3 n / 4}-x_{n-3}\right) I_{n-2}\right] } \\
& +\cdots+\sin \left(x_{3 n / 4}-x_{3 n / 4+2}\right) I_{3 n / 4+2} .
\end{aligned}
$$

But $\sin \left(x_{3 n / 4}-x_{n-3}\right)>\sin \left(x_{3 n / 4}-x_{n-1}\right)-\left(x_{n-3}-x_{n-1}\right) \cos \left(x_{3 n / 4}-x_{n-3}\right)$; $I_{n-1}+I_{n-2}>0 ; x_{n-3}-x_{n-1}<\sin (\pi / n) ; \cos \left(x_{3 n / 4}-x_{n-3}\right)<1$. Therefore,

$$
\begin{aligned}
& \int_{0}^{x_{3 n / 4}} \sin \left(x_{3 n / 4}-t\right) T_{n}^{*}(t) d t \\
&>-\sin (\pi / n) \phi(n)[\sin (2 \pi / n)+\sin (4 \pi / n)+\cdots+\sin (n / 4-1) \pi / n], \\
& \sum_{k=1}^{(n-4) / 8} \sin (2 k \pi / n)=[\cos (\pi / n)-\cos (\pi / 4)] /(2 \sin (\pi / n)) \\
& \rightarrow\left(1-2^{-1 / 2}\right) /(2 \pi / n) .
\end{aligned}
$$

Hence

$$
\int_{0}^{3 n / 4} \sin \left(x_{3 n / 4}-t\right) T_{n}^{*}(t) d t>-P(n)\left(2-2^{1 / 2}\right) / 4
$$

Therefore

$$
(-1)^{(n / 2)} e_{1}^{(n)}\left(x_{3 n / 4}\right)>P(n)\left[\left|\tau_{2}^{(n)}\right| \cos \left(\left(2-2^{1 / 2}\right) / 4\right)-\left|\tau_{1}^{(n)}\right|\left(2^{1 / 2}-1\right)\right] 2 \sqrt{2},
$$

and

$$
\left\|e_{1}^{(n)}(x)\right\|>4.259 \times 10^{-2} P(n) 2^{1-2 n} / n ! .
$$

Proof. Part (ii). An upper bound for $\left\|e_{2}^{(n)}(x)\right\|$ is obtained as before.

$$
\begin{aligned}
e_{2}^{(n)}(x)= & -\frac{\tau_{1}^{(n)}}{4}\left[\frac{T_{n+1}^{*}(x)}{n+1}-\frac{T_{n-1}^{*}(x)}{n-1}\right]+(-1)^{n}\left[\tau_{1}^{(n)} \cos x-\tau_{2}^{(n)} \sin x\right] / 2\left(n^{2}-1\right) \\
& +\int_{0}^{x} I(t)\left[\tau_{2}^{(n)} \cos (x-t)-\tau_{1}^{(n)} \sin (x-t)\right] d t, \\
\left|e_{2}^{(n)}(x)\right| \leqslant & \left\{\left[\left|\tau_{1}^{(n)}\right|(2 n-(n-1) \cos x)\right]+\left|\tau_{2}^{(n)}\right|[(n+1) \sin x]\right\} /\left[2\left(n^{2}-1\right)\right], \\
\left\|e_{2}^{(n)}\right\| & \leqslant\left[\left|\tau_{1}^{(n)}\right|(2-\cos (1))+\left|\tau_{2}^{(n)}\right| \sin (1)\right]\left[O\left(1 / n^{2}\right)+1 /(2(n+1))\right] \\
& \leqslant 1.76971(1+O(1 / n)) /\left[2^{2 n}(n+1) !\right] .
\end{aligned}
$$

To find a lower bound for $\left\|e_{2}^{(n)}(x)\right\|$ consider $e_{2}^{(n)}\left(x_{n / 2}\right)$ where $x_{n / 2} \rightarrow \frac{1}{2}$. This time

$$
\begin{aligned}
\int_{0}^{x_{n / 2}} \cos \left(x_{n / 2}-t\right) T_{n}^{*}(t) d t & >\cos \left(x_{n / 2}\right) 2 \sin (\pi / 2 n) \phi(n / 2 \pi+O(1)) \\
& \rightarrow \frac{1}{2} \cos \left(\frac{1}{2}\right) P(n)
\end{aligned}
$$


and

$$
\begin{aligned}
\int_{0}^{x_{n / 2}} \sin \left(x_{n / 2}-t\right) T_{n}^{*}(t) d t & <\sin \left(x_{n / 2}\right) 2 \sin (\pi / 2 n) \phi(n)(n / 2 \pi+O(1)) \\
& \rightarrow \frac{1}{2} \sin \left(\frac{1}{2}\right) P(n) \\
(-1)^{n / 2} e_{2}\left(x_{n / 2}\right)< & P(n)\left[\left|\tau_{2}^{(n)}\right| \sin \left(\frac{1}{2}\right)-\left|\tau_{1}^{(n)}\right| \cos \left(\frac{1}{2}\right)\right] / 2
\end{aligned}
$$

Therefore

$$
\left\|e_{2}^{(n)}(x)\right\|>0.49468 P(n) 2^{-2 n} / n ! \text {. }
$$

Remark. Since asymptotically, $P(n) \sim[1+O(1 / n)] /(n+1)$, our theorem yields:

$$
\left\|\mathrm{e}^{(n)}\right\|_{\infty}=K[1+O(1 / n)] 2^{-2 n} /(n+1) !, \quad \text { where } 0.49468<K<1.76971,
$$

if $n$ is even. This is comparable to the results of Meinardus in [4, p. 80], for the minimal deviation on $[0,1]$, except that then $K=1$.

5. The Airy Equation. We consider the form of Airy equation

$$
y^{\prime \prime}(x)+x y(x)=1, \quad \text { subject to } y(0)=A, y^{\prime}(0)=B \text {. }
$$

For $J=[0,1]$, we compute the exact solution $\left[y_{n}, z_{n}\right]^{T}$ of the perturbed system

$$
D\left[\begin{array}{l}
y_{n} \\
z_{n}
\end{array}\right]=\left[\begin{array}{cc}
x & -\frac{d}{d x} \\
\frac{d}{d x} & 1
\end{array}\right]\left[\begin{array}{l}
y_{n} \\
z_{n}
\end{array}\right]=\left[\begin{array}{l}
1 \\
0
\end{array}\right]+\tau_{1}^{(n)}\left[\begin{array}{c}
T_{n+1}^{*} \\
0
\end{array}\right]+\left[\begin{array}{cc}
\tau_{2}^{(n)} & 0 \\
0 & \tau_{3}^{(n)}
\end{array}\right]\left[\begin{array}{c}
T_{n}^{*} \\
T_{n}^{*}
\end{array}\right]
$$

The canonical polynomials associated with the matrix operator $D$ are given by the following recurrence relations:

$$
\mathbf{Q}_{k+2}^{(1)}(x)=\left[x^{k+1},-(k+1) x^{k}\right]^{T}-k(k+1) \mathbf{Q}_{k-1}^{(1)}(x),
$$

$\mathbf{Q}_{0}^{(1)}$ is undefined; $\mathbf{Q}_{1}^{(1)}=[1,0]^{T}, \mathbf{Q}_{2}^{(1)}=[x,-1]^{T}$. We note that $\mathbf{Q}_{3}^{(1)}(0)=-2 \mathbf{Q}_{0}^{(1)}(0)$; $\mathbf{Q}_{4}^{(1)}(0)=-6[1,0]^{T}$; and

$$
\begin{gathered}
\mathbf{Q}_{k+1}^{(2)}(x)=\left[(k+1) x^{k-1}, x^{k+1}\right]^{T}-(k-1)(k+1) \mathbf{Q}_{k-2}^{(2)}(x), \\
\mathbf{Q}_{0}^{(2)}=[0,1]^{T}, \quad \mathbf{Q}_{1}^{(2)}=[0, x]^{T}+\mathbf{Q}_{0}^{(1)} ; \quad \mathbf{Q}_{2}^{(2)}=\left[2, x^{2}\right]^{T} .
\end{gathered}
$$

Again, we note that $\mathbf{Q}_{3}^{(2)}(0)=-3[0,1]^{T} ; \mathbf{Q}_{4}^{(2)}(0)=-8 \mathbf{Q}_{0}^{(1)}(0)$. To obtain a Tau solution let us take

$$
\mathbf{H}_{n}(x)=\left[\tau_{1}^{(n)} T_{n+1}^{*}(x)+\tau_{2}^{(n)} T_{n}^{*}(x), \tau_{3}^{(n)} T_{n}^{*}(x)\right]^{T} .
$$

Then $\mathbf{y}_{n}(x)$ takes the form

$$
\begin{aligned}
\mathbf{y}_{n}(x)= & \mathbf{Q}_{0}^{(1)}(x)+\tau_{1}^{(n)} \sum_{k=0}^{n+1} c_{k}^{(n+1)} \mathbf{Q}_{k}^{(1)}(x)+\tau_{2}^{(n)} \sum_{k=0}^{n} c_{k}^{(n)} \mathbf{Q}_{k}^{(1)}(x) \\
& +\tau_{3}^{(n)} \sum_{k=0}^{n} c_{k} \mathbf{Q}_{k}^{(2)}(x) .
\end{aligned}
$$

We shall employ an extra condition to make the coefficient of $\mathbf{Q}_{0}^{(1)}(x)$ identically equal to zero in the expression of $y_{n}(x)$. 
For example, let $n=3, A=3^{-2 / 3} \Gamma(1 / 3), B=-3^{-1 / 3} \Gamma(2 / 3)$. The tau-terms in the expression of $\mathbf{y}_{n}(x)$ follow from the three conditions: $\mathbf{y}_{n}(0)=[A, B]^{T}$, and the cancellation of the coefficient of $\mathbf{Q}_{0}^{(1)}(x)$ in $\mathbf{y}_{n}(x)$ :

$$
1+\tau_{1}^{(3)}\left[c_{0}^{(4)}-2 c_{3}^{(4)}\right]+\tau_{2}^{(3)}\left[c_{0}^{(3)}-2 c_{3}^{(3)}\right]+\tau_{3}^{(3)}\left[c_{1}^{(3)}\right]=0
$$

Hence we find

$$
\tau_{3}^{(3)}=0.011766 ; \quad \tau_{2}^{(3)}=-0.006330 ; \quad \tau_{1}^{(3)}=-0.003164
$$

Let us now develop our Tau solution analytically, using the Green's function. Two linearly independent solutions of the homogeneous version of (19) are given by

$$
u(x)=x^{1 / 2} J_{1 / 3}\left(2 x^{3 / 2} / 3\right), \quad v(x)=x^{1 / 2} J_{-1 / 3}\left(2 x^{3 / 2} / 3\right) .
$$

Let $k(x, t), W(t)$ and $\phi(x, t)$ be defined as before. From the relationships

$$
J_{p}^{\prime}(t)=J_{p-1}(t)-(p / t) J_{p}(t), \quad J_{p}^{\prime}(t)=(p / t) J_{p}(t)-J_{p+1}(t)
$$

we obtain, respectively,

$$
\begin{gathered}
u^{\prime}(x)=x J_{-2 / 3}\left(2 x^{3 / 2} / 3\right), \quad v^{\prime}(x)=-x J_{2 / 3}\left(2 x^{3 / 2} / 3\right), \\
u^{\prime \prime}(x)=-x^{3 / 2} J_{1 / 3}\left(2 x^{3 / 2} / 3\right), \quad v^{\prime \prime}(x)=-x^{3 / 2} J_{1 / 3}\left(2 x^{3 / 2} / 3\right) .
\end{gathered}
$$

From the series expansion of the Bessel functions we have

$$
\begin{gathered}
u(0)=0, \quad v(0)=3^{1 / 3} / \Gamma(2 / 3), \quad u^{\prime}(0)=3^{2 / 3} / \Gamma(1 / 3), \quad v^{\prime}(0)=0, \\
W(0)=-3 /[\Gamma(1 / 3) \Gamma(2 / 3)] \quad \text { and } W(t)=W(0) \text { for all } t
\end{gathered}
$$

$y_{n}(x), z_{n}(x)$ are the solutions, respectively, of

$$
\begin{aligned}
y_{n}^{\prime \prime}(x)+x y_{n}(x) & =1+\tau_{1}^{(n)} T_{n+1}^{*}(x)+\tau_{2}^{(n)} T_{n}^{*}(x)+\tau_{3}^{(n)} T_{n}^{*^{\prime}}(x), \\
z_{n}^{\prime}(x) & =x y_{n}(x)-1-\tau_{1}^{(n)} T_{n+1}^{*}(x)-\tau_{2}^{(n)} T_{n}^{*}(x) .
\end{aligned}
$$

The solution of $(21)$ is

$$
y_{n}(x)=c_{1} u(x)+c_{2} v(x)-\tau_{3}^{(n)} T_{n}^{*}(0) \phi(x, 0)+\int_{0}^{x} \frac{G(x, t)}{W(0)} d t,
$$

where

$$
G(x, t)=\left[1+\tau_{1}^{(n)} T_{n+1}^{*}(t)+\tau_{2}^{(n)} T_{n}^{*}(t)\right] k(x, t)-\tau_{3}^{(n)} T_{n}^{*}(t) \frac{\partial}{\partial t} k(x, t) .
$$

We deduce, as in Section 2, that

$$
y_{n}(x)-y(x)=\int_{0}^{x} \frac{G(x, t)-k(x, t)}{W(0)} d t .
$$

On the other hand, one readily obtains from (22)

$$
z_{n}(x)-z(x)=\int_{0}^{x} t\left[y_{n}(t)-y(t)\right] d t-\int_{0}^{x}\left[\tau_{1}^{(n)} T_{n+1}^{*}(t)+\tau_{2}^{(n)} T_{n}(t)\right] d t
$$

We now find upper bounds for the error function in terms of the tau-terms. From the expansion $J_{p}(z)=|z / 2|_{0}^{p} F_{1}\left(p+1 ;-z^{2} / 4\right) / \Gamma(p+1)$ we obtain, for real $t$, 


$$
\begin{gathered}
p>-1 ;\left|J_{p}(t)\right| \leqslant|t / 2|^{p} / \Gamma(p+1) . \text { Hence for } 0 \leqslant x \leqslant 1 \\
|u(x)| \leqslant(1 / 3)^{1 / 3} / \Gamma(4 / 3) ; \quad|v(x)| \leqslant(1 / 3)^{-1 / 3} / \Gamma(2 / 3) ; \\
\left|u^{\prime}(x)\right| \leqslant(1 / 3)^{-2 / 3} / \Gamma(1 / 3) ; \quad\left|v^{\prime}(x)\right| \leqslant(1 / 3)^{2 / 3} / \Gamma(5 / 3) ; \\
|k(x, t)| \leqslant 2 /[\Gamma(2 / 3) \Gamma(4 / 3)]=2|W(0)| ; \\
\left|k_{t}(x, t)\right| \leqslant 9 /[2 \Gamma(1 / 3) \Gamma(2 / 3)]=3|W(0)| / 2 ; \quad\left|k_{t t}(x, t)\right| \leqslant 2|W(0)| ;
\end{gathered}
$$

and

$$
\begin{aligned}
& \left\|y_{n}-y\right\| \leqslant(3 / 4 n)\left|\tau_{1}^{(n)}\right|+(3 / 4(n-1))\left|\tau_{2}^{(n)}\right|+(3 / 2(n-1))\left|\tau_{3}^{(n)}\right|, \\
& \left\|z_{n}-z\right\| \leqslant(7 / 8 n)\left|\tau_{1}^{(n)}\right|+(7 / 8(n-1))\left|\tau_{2}^{(n)}\right|+(3 / 4(n-1))\left|\tau_{3}^{(n)}\right| .
\end{aligned}
$$

Hence we find, for $n=3$, the following bounds for the components of the error function:

$$
\left\|y_{3}-y\right\|<0.01199, \quad\left\|z_{3}-z\right\|<0.008105,
$$

in agreement with the computed values.

Department of Mathematics

Imperial College, University of London

London, England

1. J. H. FreIlich \& E. L. Ortiz, Simultaneous Approximation of a Function and its Derivative with the Tau Method, Conf. on Numerical Analysis, Dundee, 1975 and Imperial College, NAS Res. Report, 1975, pp. 1-45.

2. C. Lanczos, "Trigonometric interpolation of empirical and analytical functions," J. Math. Phys., v. 17, 1938, pp. 123-199.

3. Y. L. Luke, The Special Functions and Their Approximations, Vols. I and II, Academic Press, New York, 1969.

4. G. MeInARDUS, Approximation of Functions: Theory and Numerical Methods, Springer-Verlag, Berlin, 1967.

5. E. L. Ortiz, “The Tau method,” SIAM J. Numer. Anal., v. 6, 1969, pp. 480-492.

6. E. L. ORTIZ, "On the numerical solution of nonlinear and functional differential equations with the Tau method," in Numerical Treatment of Differential Equations in Applications (R. Ansorge and W. Törnig, Eds.), Springer-Verlag, Berlin and New York, 1978, pp. 127-139.

7. E. L. Ortiz, “Step by step Tau method," Comput. Math. Appl., v. 1, 1975, pp. 381-392.

8. E. L. ORTiz \& H. Samara, "A new operational approach to the numerical solution of differential equations in terms of polynomials," in Innovative Numerical Analysis for the Engineering Sciences (R. Shaw and W. Pilkey, Eds.), The University Press of Virginia, Charlottesville, Va., 1980, pp. 643-652. 